\title{
Expression, purification and production of antisera against recombinant truncated VP22 protein
}

\author{
XIAN YU ${ }^{1}$, JUN LEI $^{2}$, QIN YANG ${ }^{2}$, ZHENGMIN XU $^{2}$ and YAN WANG $^{2}$ \\ ${ }^{1}$ Department of Pharmacy, The Second Affiliated Hospital of Chongqing Medical University, Chongqing 400010; \\ ${ }^{2}$ Institute of Materia Medica, North Sichuan Medical College, Nanchong, Sichuan 637007, P.R. China
}

Received December 4, 2014; Accepted February 11, 2016

DOI: $10.3892 /$ etm.2016.3103

\begin{abstract}
Cell-penetrating peptides (CPPs) are non-invasive vectors that can efficiently transport bioactive cargo across the cell membrane. Naturally occurring CPPs, such as the tegument protein VP22 of the Herpes simplex virus type 1, can potentiate protein-drug delivery into living cells. The aim of the present study was to construct anti-VP22 antibodies that can be used to detect VP22-fusion drugs. Therefore, 60- and 45-amino acid peptides corresponding to the $\mathrm{N}$-terminus and C-terminus of VP22, respectively, were cloned, expressed and purified. Subsequently, polyclonal antisera against them were generated. The DNA sequence, cloned into the pGEX-5X-1 vector, was transformed into $E$. coli BL21 (DE3). After inducing expression with $1 \mathrm{mM}$ isopropyl- $\beta$-D-thiogalactopyra noside (IPTG) at $25^{\circ} \mathrm{C}$ for $4 \mathrm{~h}$, the recombinant VP22 proteins were purified by electroelution. The high titers of polyclonal antisera obtained subsequent to immunization of mice with the purified recombinant truncated VP22 was confirmed by ELISA. Western blot and immunofluorescence analysis showed that the antisera detected both the truncated and full-length VP22 protein. Therefore, the polyclonal antisera against VP22 may be used in the detection of the intracellular location of VP22-fusion protein drugs.
\end{abstract}

\section{Introduction}

Cell-penetrating peptides (CPPs), also known as protein transduction domains (1) or Trojan peptides, are able to overcome limitations of large molecular (DNA and proteins) drug delivery systems (viral and non-viral) commonly used currently, including membrane penetration and toxicity (2). CPPs non-invasively translocate through the cell membrane to ubiquitously deliver a wide-range of hydrophilic and hydrophobic bioactive molecules, including proteins, DNA

Correspondence to: Dr Xian Yu, Department of Pharmacy, The Second Affiliated Hospital of Chongqing Medical University, 76 Linjiang Road, Chongqing 400010, P.R. China

E-mail: xiannyyu@gmail.com

Key words: antisera, expression, purification, VP22 and liposomes; thus, CPPs can be used as a versatile tool for intracellular delivery (3). Although the exact mechanisms underlying CPP internalization are unclear, it is speculated that various endocytic pathways and direct membrane translocation are involved (4).

The tegument protein VP22 of the Herpes simplex virus type 1 (HSV-1) is a naturally occurring CPP with an effective intracellular spread $(5,6)$. The recombinant VP22 protein augments the therapeutic effects of protein drugs (7), as well as of gene and immune therapies $(8,9)$, by increasing the intracellular distribution of therapeutic molecules. VP22 fusion-proteins are currently in use for the treatment of diseases such as congestive heart failure and C6 glioma $(8,10)$. The aim of the present study was to provide novel molecular tools to assist advancement in the development of VP22-fusion therapeutics. Thus, the expression of peptides corresponding to the N-terminus and C-terminus of VP22 were detected, and subsequently antisera against these peptides were produced, which detected both the truncated and the full-length VP22 protein.

\section{Materials and methods}

Plasmids and strains. pcDNA3-VP22 expressing HSV 1 VP22 was constructed as described previously (11). Glutathione S-transferase (GST)-tagged pGEX-5X-1 (GE Healthcare Life Sciences, Little Chalfont, UK) vector acted as prokaryotic expression vectors. E. coli BL21 (DE3) cells (Invitrogen; Thermo Fisher Scientific, Inc., Waltham, MA, USA) were used for inducible protein expression.

Cells. Vero cells (Laboratory of Biochemistry and Molecular Pharmacology, Chongqing Medical University, Chongqing, China) were used for verification of polyclonal antibody binding.

Experimental animals. A total of 20 female BALB/c mice (age, 4-6 weeks; weight, $15 \mathrm{~g}$; Animal Laboratory Center of Chongqing Medical University) were maintained in specific pathogen-free, environmentally controlled conditions at $22 \pm 2{ }^{\circ} \mathrm{C}$ with $50-70 \%$ humidity. Animals had ad libitum access to food and water. The use of animals and the experimental protocols were approved by the Ethics Committee of Chongqing Medical University. 
Table I. Primers for the construction of recombinant plasmids.

\begin{tabular}{lcl}
\hline Primer & Restriction site & Sequence (5' to 3') \\
\hline N-terminal 60 aa forward & $E c o$ RI & ATAGAATTCATGACCTCTCGCCGC \\
N-terminal 60 aa reverse & XhoI & ATTCTCGAGGTACTGGACGAAACG \\
C-terminal 45 aa forward & Bam $\mathrm{HI}$ & ATCGGATCCAAGAGTTGGTGAATCCA \\
C-terminal 45 aa reverse & EcoRI & ATTGAATTCTCACTCGACGGGCCG
\end{tabular}

aa, amino acids.

Cloning of VP22 peptides into the $p G E X-5 X-1$ vector. A pcDNA3-VP22 containing the HSV-1 VP22 gene was constructed as described previously (11). Briefly, the HSV-1 VP22 gene was amplified using polymerase chain reaction (PCR) from a HSV-1 virus harvest. The VP22 amplicon was digested with EcoRI (Takara Biotechnology Co., Ltd., Dalian, China) and subcloned into a eukaryotic expression vector pcDNA3 (Invitrogen; Thermo Fisher Scientific, Inc.), resulting in pcDNA3-VP22. Using pcDNA3-VP22 as a template, the DNA sequence expressing the first 60 amino acids (aa) of the $\mathrm{N}$-terminus and the last 45 aa of the C-terminus of VP22 were amplified by PCR. The primers and restriction enzymes used for recombination are listed in Table I. PCR was performed on GeneAmp 2400 PCR system (PerkinElmer, Boston, MA, USA), with an initial denaturation at $94^{\circ} \mathrm{C}$ for $3 \mathrm{~min}$, followed by 30 cycles of denaturation at $94^{\circ} \mathrm{C}$ for $30 \mathrm{sec}$, annealing at $55^{\circ} \mathrm{C}$ for $30 \mathrm{sec}$, extension at $72^{\circ} \mathrm{C}$ for $30 \mathrm{sec}$, and a final extension at $72^{\circ} \mathrm{C}$ for $10 \mathrm{~min}$. The amplified genes were gel-purified using an agarose gel extraction kit (Tiangen Biotech Co., Ltd., Beijing, China), according to the manufacturer's instructions. Double restriction endonuclease digestion using EcoRI/XhoI (Takara Biotechnology Co., Ltd.) was performed in pGEX-5X-1 and the PCR fragment expressing N-terminal 60 aa of VP22. Double restriction endonuclease digestion using BamHI/EcoRI (Takara Biotechnology Co., Ltd.) was performed in pGEX-5X-1 and the PCR fragment expressing the C-terminal 45 aa of VP22. The digested target fragments and vectors were ligated at $16^{\circ} \mathrm{C}$ for $1 \mathrm{~h}$ using a T4 DNA ligase kit (Takara Biotechnology Co., Ltd.) resulting in pGEX-N60 (expressing GST-tagged N-terminal 60 aa of VP22) or pGEX-C45 (expressing GST-tagged C-terminal 45 aa of VP22). The recombinant vectors were identified by restriction analysis with $E c o \mathrm{RI} / X h o \mathrm{I}$ or $B a m \mathrm{HI} / E c o \mathrm{RI}$ at $37^{\circ} \mathrm{C}$ for $2 \mathrm{~h}$ and DNA sequencing by Sangon Biotech Co., Ltd. (Shanghai, China), after which they were transformed into competent E. coli BL21 (DE3) cells.

Expression of recombinant VP22 proteins. E. coli BL21 (DE3) cells were chemically transformed with pGEX-N60 or pGEX-C45, and grown overnight in Luria-Bertani medium (Sigma-Aldrich, St. Louis, MO, USA) containing $100 \mu \mathrm{g} / \mathrm{ml}$ ampicillin (Tiangen Biotech Co., Ltd.) at $37^{\circ} \mathrm{C}$. Next, $0.5 \mathrm{ml}$ of the overnight $E$. coli cell culture was transferred into fresh medium in a culture flask and grown until an optical density at $600 \mathrm{~nm}$ of 0.5 was reached (SP-756 UV-Vis Spectrophotometer; Shanghai Spectrum Instrument Co., Ltd., Shanghai, China). Subsequently, expression of the recombinant protein was induced by addition of $1 \mathrm{mM}$ isopropyl $\beta$-D-1-thiogalactopyranoside (IPTG) for $4 \mathrm{~h}$ at $25^{\circ} \mathrm{C}$.

Extraction of recombinant VP22 protein. E. coli BL21 (DE3) expressing cells were collected by centrifugation at 4,000 x g for $15 \mathrm{~min}$ at room temperature. The cell pellet was washed three times with double-distilled (dd) $\mathrm{H}_{2} \mathrm{O}$ and incubated in $5 \mathrm{ml}$ lysis buffer $[50 \mathrm{mM}$ phosphate-buffered saline (PBS), $\mathrm{pH} 7.4 ; 0.5 \mathrm{M} \mathrm{NaCl} ; 1 \mathrm{mM} \mathrm{MgCl}$; $0.5 \mathrm{mg} / \mathrm{ml}$ lysozyme; and $1 \mathrm{mM}$ phenylmethylsulfonyl fluoride] on ice for $45 \mathrm{~min}$. Next, the cells were sonicated at $50 \%$ duty cycle and $300 \mathrm{~W}$ for $8 \mathrm{~min}$ using an ultrasonic disintegrator (scientz-IID; Ningbo Xinzhi Instruments Inc., Ningbo, China). The soluble fraction was then collected following centrifugation at $15,000 \mathrm{x}$ g for $10 \mathrm{~min}$ at $4^{\circ} \mathrm{C}$. The inclusion bodies (insoluble fraction) were dissolved in $2 \mathrm{ml}$ urea $(6 \mathrm{M})$, with incubation at $42^{\circ} \mathrm{C}$ for $30 \mathrm{~min}$, and then recovered by centrifugation at $8,000 \mathrm{x} \mathrm{g}$ for $10 \mathrm{~min}$ at room temperature. Subsequently, the soluble fraction and the dissolved inclusion bodies were subjected to $12 \%$ SDS-PAGE and Coomassie Brilliant Blue R250 (Beyotime Institute of Biotechnology, Haimen, China) staining to determine the protein expression.

Purification of recombinant VP22 proteins by electroelution. The excised recombinant protein bands were subjected to electroelution at $4^{\circ} \mathrm{C}$ for $3 \mathrm{~h}$ at $100 \mathrm{~mA}$, using an electroelution buffer (25 mM Tris-HCl, $250 \mathrm{mM}$ glycine and 0.1\% SDS; $\mathrm{pH}$ 8.3) and a dialysis bag (Sangon Biotech Co., Ltd.) with a 6-kDa molecular weight cut-off. Electroelution was terminated when the Coomassie Brilliant Blue R250 dye completely ran from the SDS-PAGE gels into electroelution completely, and the electroelution was incubated in five volumes of acetone at $-20^{\circ} \mathrm{C}$ for $1 \mathrm{~h}$. The recombinant protein pellet was harvested by centrifugation at $12,000 \mathrm{x}$ g for $20 \mathrm{~min}$ at $4^{\circ} \mathrm{C}$, dissolved in $\mathrm{ddH}_{2} \mathrm{O}$ and desalted by running it through a Sephadex G-25 column (GE Healthcare Life Sciences) to remove excess small molecules (12).

Production and purification of polyclonal antisera against the recombinant proteins. Antibodies against each recombinant VP22 protein were obtained by immunizing 4-6-week-old female BALB/c mice. Each animal was initially injected subcutaneously with $50 \mu \mathrm{g}$ purified recombinant protein emulsified in Freund's complete adjuvant (1:1; Sigma-Aldrich), subsequent to collecting pre-immune sera. Two booster injections of $0.5 \mathrm{mg} / \mathrm{ml}$ recombinant protein in equal volume of incomplete Freund's adjuvant (Sigma-Aldrich) were given at 


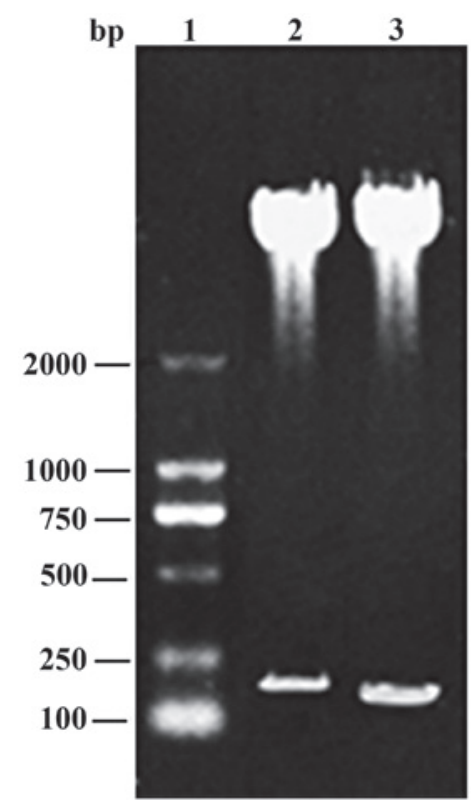

Figure 1. Restriction analysis of recombinant plasmids DNA ladder (lane 1); pGEX-N60 identified by digestion with EcoRI/XhoI (lane 2); and pGEX-C45 identified by BamHI/EcoRI digestion (lane 3).

2-week intervals in order to obtain a prolonged persistence of the immunogen in the tissues and a continuous stimulation of the immune system. At 10 days after the final injection, blood was collected from the tail vein of the immunized mice, and the crude antisera were collected by centrifugation at $4,200 \mathrm{xg}$ for $5 \mathrm{~min}$ at room temperature. The immunoglobulin (Ig) antibodies were precipitated with ammonium sulfate (40\% saturation) and dissolved in $2 \mathrm{ml}$ of $0.2 \mathrm{M} \mathrm{NaCl}$.

ELISA validation of the polyclonal antisera. ELISA plates (Corning, Inc., Corning, NY, USA) were coated with $100 \mu 1$ purified recombinant VP22 proteins $(5 \mu \mathrm{g} / \mathrm{ml})$ in $50 \mathrm{mM}$ carbonate-bicarbonate buffer $(\mathrm{pH} 9.6)$ and incubated at $4^{\circ} \mathrm{C}$ overnight. Subsequent to washing with $0.5 \%$ PBS-Tween-20 (pH 7.6), non-specific proteins were blocked with $150 \mu \mathrm{l}$ $5 \%$ bovine serum albumin for $1 \mathrm{~h}$ at room temperature and then incubated with $100 \mu \mathrm{l}$ polyclonal antiserum of different dilutions (between 1:200 and 1:64,000) for $2 \mathrm{~h}$ at $37^{\circ} \mathrm{C}$. Following incubation with $100 \mu \mathrm{l}$ of horseradish peroxidase (HRP)-conjugated goat anti-mouse IgG (Santa Cruz Biotechnology, Inc., Santa Cruz, CA, USA; 1:2,000; sc-2005) for $1 \mathrm{~h}$ at $37^{\circ} \mathrm{C}$, the proteins were detected using a TMB Detection system (Wuhan Boster Biological Technology, Ltd., Wuhan, China), and binding was quantified by measuring the absorbance at $450 \mathrm{~nm}$ with SpectraMax 190 microplate reader (Molecular Devices LLC, Sunnyvale, CA, USA). The pre-immunized mouse serum served as a negative control.

Western blot analysis. Vero cells grown to $70 \%$ confluence in 6-well plates and were transfected with pcDNA3-VP22 (the full length VP22), pcDNA3-VP22-N (the first 150 aa of the VP22 N-terminus), or pcDNA3-VP22-C (the last 151 aa of the VP22 C-terminus). After $48 \mathrm{~h}$, the cells were lysed in radioimmunoprecipitation assay buffer (Bryotime Institute of Biotechnology), and the proteins were subjected
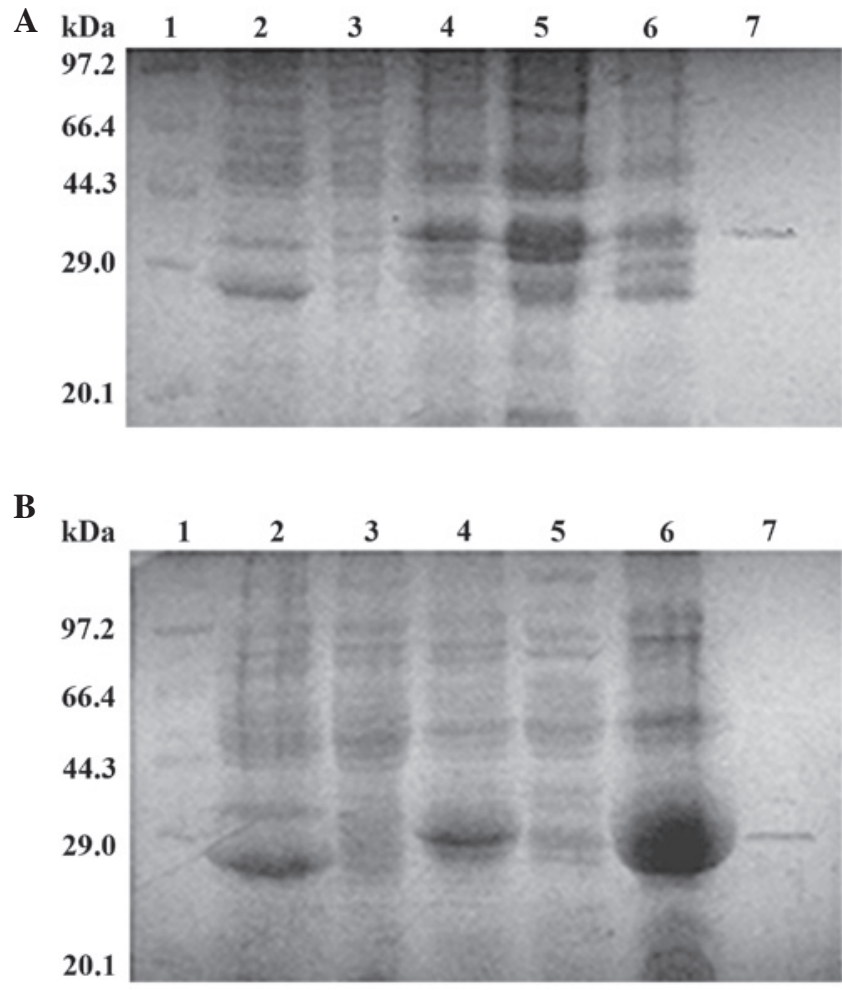

Figure 2. Expression and purification of truncated VP22 analyzed by SDS-PAGE. (A) Gel showing the following: Protein standard (lane 1); total protein from induced pGEX-5X-1 (lane 2); non-induced pGEX-N60 (lane 3) and induced pGEX-N60 (lane 4) transformed E. coli; soluble (lane 5) and insoluble (lane 6) fractions from induced pGEX-N60 transformed E. coli; and purified recombinant N60-GST protein (lane 7). (B) Gel showing the following: Protein standard (lane 1); total protein from induced pGEX-5X-1 (lane 2), non-induced pGEX-C45 (lane 3), and induced pGEX-C45 (lane 4) transformed E. coli; soluble (lane 5) and insoluble (lane 6) fractions from induced pGEX-C45 transformed E. coli; and purified recombinant C45-GST protein (lane 7).

to $12 \%$ SDS-PAGE and then electrotransferred to a polyvinylidene difluoride membrane. Incubation with the purified anti-recombinant VP22 (1:500) was followed by incubation with secondary HRP-conjugated goat anti-mouse IgG (Santa Cruz Biotechnology, Inc.; 1:2,000; sc-2005), and the proteins were detected using the DAB Detection Kit (Wuhan Boster Biological Technology, Ltd.). The empty pcDNA3-transfected cells served as a negative control.

Immunofluorescence assay. Vero cells were grown to $70 \%$ confluence on glass coverslips and transfected with the pcDNA3-VP22 plasmid. At $48 \mathrm{~h}$ post-transfection, the cells were washed with PBS, fixed in cold methanol for $10 \mathrm{~min}$ at room temperature, and then permeabilized with $0.2 \%$ Triton X-100-PBS (Sigma-Aldrich) for $90 \mathrm{~min}$ at room temperature. Following a wash with PBS, the cells were blocked for $30 \mathrm{~min}$ in 5\% non-fat milk at room temperature and incubated with anti-recombinant VP22 sera $(1: 100)$ at $4{ }^{\circ} \mathrm{C}$ overnight. Subsequent to three further washes with PBS, the cells were incubated with fluorescein isothiocyanate-conjugated goat anti-mouse IgG (Santa Cruz Biotechnology, Inc.; 1:1,000; sc-2010) for $1 \mathrm{~h}$ at room temperature and observed by inverted fluorescence microscopy (TCS SP2; Leica Microsystems, Wetzlar, Germany). The pre-immunized mouse serum served as negative control. 
$\mathbf{A}$

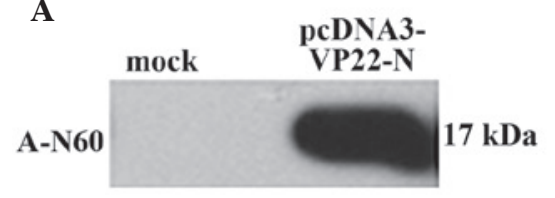

B

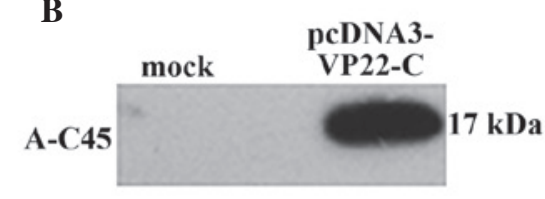

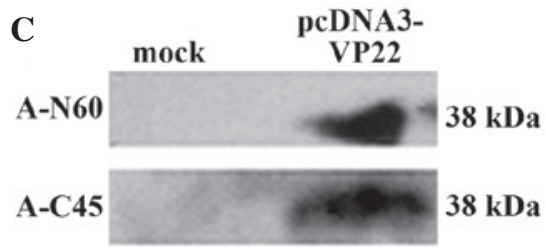

Figure 3. Western blot analysis of antiserum against recombinant truncated VP22 proteins. (A) VP22 N-terminal 150 aa and (B) VP22 C-terminal 151 aa were detected with the specific antisera, anti-N60 and anti-C45, respectively. (C) Full-length VP22 protein was detected with anti-N60 and anti-C45. Mock represents pcDNA3-transfected cells as the negative control. aa, amino acid.
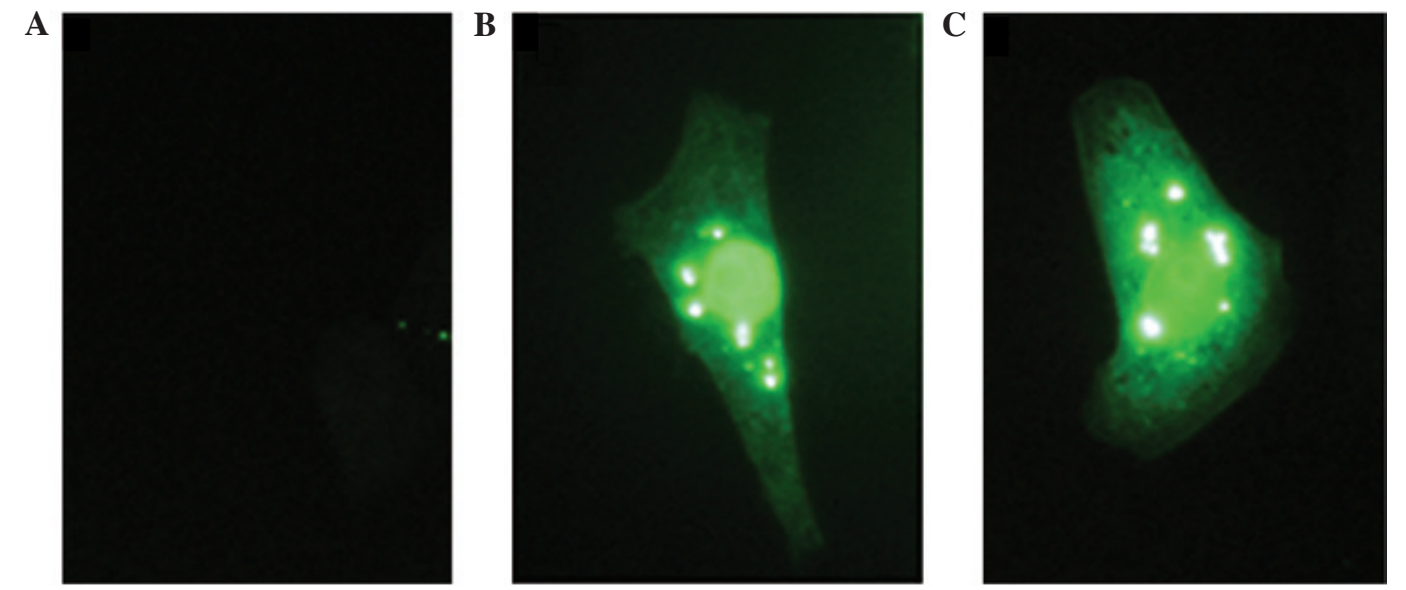

Figure 4. Immunofluorescence detection of the full-length VP22 protein using antibodies against recombinant truncated VP22 proteins. (A) Pre-immunized mouse serum was not able to detect the full-length VP22 protein (negative control). (B) Anti-N60 and (C) anti-C45 were able to detect the full-length VP22 protein in the cytoplasm and the nucleus of Vero cells. Magnification, x60.

\section{Results}

Expression vector construction. The PCR-amplified $\mathrm{N}$-terminal (60 aa) and C-terminal (45 aa) DNA sequences corresponded with the expected sizes of $180 \mathrm{bp}$ and $135 \mathrm{bp}$, respectively. The PCR products were ligated into the pGEX-5X-1 vector and transformed into competent E. coli BL21 (DE3) cells, and the positive clones were identified by restriction analysis (Fig. 1). DNA sequencing confirmed that pGEX-N60 and pGEX-C45 were constructed.

Expression and purification of the recombinant proteins. IPTG addition induced the expression of recombinant proteins in the E. coli BL21 (DE3) transformants. Since the molecular weight of GST is $26 \mathrm{kDa}$, the expected molecular weights for GST-tagged N-terminal 60 aa of VP22 (N60-GST) and GST-tagged C-terminal 45 aa of VP22 (C45-GST) were $32 \mathrm{kDa}$ and $30 \mathrm{kDa}$, respectively. N60-GST and C45-GST migrated to a position in the gel close to the $29 \mathrm{kDa}$ band in the molecular weight markers and were close to the expected molecular weights (Fig. 2A and B, lanes 4-6). In addition, the target protein was almost absent in the non-induced transformants (Fig. 2A and B, lane 3). Upon analyzing the soluble fraction and inclusion bodies, the recombinant protein N60-GST was found to circulate in the soluble fraction (Fig. 2A, lane 5), while the majority of the recombinant protein C45-GST was present in the inclusion bodies (Fig. 2B, lane 6). Since the recombinant protein C45-GST was present in the insoluble fraction, the N60-GST and C45-GST proteins were purified by electroelution, obtaining at least $80 \mathrm{mg} / 100 \mathrm{ml}$ of each recombinant protein subsequent to desalting using the Sephadex G-25 column (Fig. 2A and B, lane 7).

Production of polyclonal antisera against recombinant VP22 proteins. The antisera against VP22 N-terminal peptide (labeled as anti-N60) and VP22 C-terminal peptide (labeled as anti-C45) were collected from mice following three antigen injections. ELISA and western blot analysis were used to validate the reactivity and specificity of the antisera, respectively. The antisera reacted at different dilutions (anti-N60 dilutions, 1:200 to 1:16,000; and anti-C45 dilutions, 1:200 to $1: 32,000)$ with an equal amount of the corresponding recombinant proteins (data not shown). No positive signal was detected from the pre-immunized mouse serum, which acted as a negative control. The results indicated that anti-N60 and anti-C45 were able to specifically detect their respective truncated VP22 proteins (Fig. 3A and B) and full-length VP22 proteins (Fig. 3C). In addition, the antisera did not show any non-specific reaction against the pcDNA3-transfected cell lysates. The molecular weight of the N-terminal 150 aa, the C-terminal 151 aa and the full-length VP22 protein were confirmed to be approximately $17 \mathrm{kDa}, 17 \mathrm{kDa}$ and $38 \mathrm{kDa}$, respectively.

To validate the different uses of the polyclonal antibody investigated in the present study, the subcellular localization of VP22 protein in pcDNA3-VP22-transfected Vero cells by immunofluorescence was examined. Pre-immunized mouse 
serum was used as the negative control (Fig. 4A). The results indicated that anti-N60 (Fig. 4B) and anti-C45 (Fig. 4C) detected the full-length VP22, while they showed medium cytosolic and predominant nuclear localization of VP22 in the Vero cells. In conclusion, in the present study, polyclonal antisera against the $\mathrm{N}$ - and $\mathrm{C}$-terminus of VP22 that recognizes the truncated and full-length VP22 protein were developed. These antisera may serve as a useful tool for further studies of VP22-fusion protein drugs, and facilitate the development of these protein drugs in future studies.

\section{Discussion}

CPP-based intracellular delivery is one of the most powerful techniques used for importing protein drugs into eukaryotic cells $(13,14)$. However, the lack of tools to track VP22-fusion therapeutic molecules in cells may be a limitation in the development of novel VP22-based treatments. The antibodies generated against the $\mathrm{C}$ - and $\mathrm{N}$-terminus of the VP22 protein may be used to identify the cellular location of the VP22-fusion drugs. The C-terminus of VP22 protein is essential for intercellular transport $(9,15)$, and the polyclonal C-terminal antibody may be used to detect the position of C-terminal VP22-fusion protein drugs in the cells. The truncated recombinant proteins were cloned in frame with the N-terminal GST-tag of the pGEX-5X-1 vector.

Antibodies against different specific regions of the VP22 proteins are increasingly used in the development of VP22 fusion protein drugs $(7,16)$. In the present study, antisera against the truncated VP22 peptides recognized their specific targets and a full-length VP22 protein. These antisera can be used in various molecular applications, including ELISA, western blot analysis and immunofluorescence. Notably, the molecular weights of N60-GST, C45-GST, N-terminal 150 aa and C-terminal 151 aa of VP22 were consistent with the calculated theoretical values. The molecular weight of the full-length VP22 protein was consistent with that of the basic phosphorylated VP22 (17). Furthermore, the cytosolic and nuclear localization of VP22 was consistent with that reported previously (18), thus further validating the antisera.

Currently, a limitation of large molecular drug therapy (with genes or proteins) is the inability to deliver sufficient amounts of active large molecular drugs to the target cells (19). While secreted proteins can overcome this limitation to a certain degree, this is challenging for non-secreted proteins. In these cases, the potentially therapeutic protein is only active in cells to which it is initially delivered. It has been suggested that VP22 fusion proteins may be able to provide a solution by increasing drug distribution through intercellular delivery $(8,20)$. Theoretically, more cells would then reach a therapeutic steady-state, leading to an overall enhancement of biological effects in the target population. Further studies should investigate the antitumor activity of VP22-fused antitumor protein drugs and evaluate the immune response of VP22-fused antigens.

In conclusion, in the present study, polyclonal antisera against VP22 were developed. These antisera will facilitate the development of VP22-fusion protein drugs in future studies.

\section{Acknowledgements}

This study was supported by a grant from the National Natural Science Foundation of China (no. 81102288) and Chongqing Science \& Technology Commission (no. cstc2014jcyjA10009).

\section{References}

1. Zahid M and Robbins PD: Cell-type specific penetrating peptides: Therapeutic promises and challenges. Molecules 20: 13055-13070, 2015.

2. Gautam A, Singh H, Tyagi A, Chaudhary K, Kumar R, Kapoor P and Raghava GP: CPPsite: A curated database of cell penetrating peptides. Database (Oxford) 2012: bas015, 2012.

3. Madani F, Lindberg S, Langel U, Futaki S and Gräslund A: Mechanisms of cellular uptake of cell-penetrating peptides. J Biophys 2011: 414729, 2011.

4. Jiao CY, Delaroche D, Burlina F, Alves ID, Chassaing G and Sagan S: Translocation and endocytosis for cell-penetrating peptide internalization. J Biol Chem 284: 33957-33965, 2009.

5. Elliott $\mathrm{G}$ and $\mathrm{O}$ 'Hare P: Intercellular trafficking and protein delivery by a herpesvirus structural protein. Cell 88: 223-233, 1997.

6. Tanaka M, Kato A, Satoh Y,Ide T, Sagou K, Kimura K, Hasegawa H and Kawaguchi Y: Herpes simplex virus 1 VP22 regulates translocation of multiple viral and cellular proteins and promotes neurovirulence. J Virol 86: 5264-5277, 2012.

7. Zavaglia D, Favrot MC, Eymin B, Tenaud C and Coll JL: Intercellular trafficking and enhanced in vivo antitumour activity of a non-virally delivered P27-VP22 fusion protein. Gene Ther 10: 314-325, 2003.

8. Jin G, Zhou Y, Chai Q, Zhu G, Xu F and Liu F: VP22 and cytosine deaminase fusion gene modified tissue-engineered neural stem cells for glioma therapy. J Cancer Res Clin Oncol 139: 475-483, 2013.

9. Nishikawa M, Otsuki T, Ota A, Guan X, Takemoto S, Takahashi Y and Takakura Y: Induction of tumor-specific immune response by gene transfer of Hsp70-cell-penetrating peptide fusion protein to tumors in mice. Mol Ther 18: 421-428, 2010.

10. Bian J, Popovic ZB, Benejam C, Kiedrowski M, Rodriguez LL and Penn MS: Effect of cell-based intercellular delivery of transcription factor GATA4 on ischemic cardiomyopathy. Circ Res 100: 1626-1633, 2007.

11. Yu X, Liu L, Wu L, Wang L, Dong C, Li W and Li Q: Herpes simplex virus type 1 tegument protein VP22 is capable of modulating the transcription of viral TK and $\mathrm{gC}$ genes via interaction with viral ICP0. Biochimie 92: 1024-1030, 2010.

12. Wang Z, Feng S, Huang Y, Qiao M, Zhang B and Xu H: Prokaryotic expression, purification and polyclonal antibody production of a hydrophobin from Grifola frondosa. Acta Biochim Biophys Sin (Shanghai) 42: 388-395, 2010.

13. Bolhassani A: Potential efficacy of cell-penetrating peptides for nucleic acid and drug delivery in cancer. Biochim Biophys Acta 1816: 232-246, 2011.

14. Sawant $\mathrm{R}$ and Torchilin V: Intracellular transduction using cell-penetrating peptides. Mol Biosyst 6: 628-640, 2010.

15. Aints A, Güven H, Gahrton G, Smith CI and Dilber MS: Mapping of herpes simplex virus-1 VP22 functional domains for inter-and subcellular protein targeting. Gene Ther 8: 1051-1056, 2001.

16. Perkins SD, Flick-Smith HC, Garmory HS, Essex-Lopresti AE, Stevenson FK and Phillpotts RJ: Evaluation of the VP22 protein for enhancement of a DNA vaccine against anthrax. Genet Vaccines 3: 3, 2005.

17. Mouzakitis G, McLauchlan J, Barreca C, Kueltzo L and O'Hare P: Characterization of VP22 in Herpes simplex virus-infected cells. J Virol 79: 12185-12198, 2005.

18. Yedowitz JC, Kotsakis A, Schlegel EF and Blaho JA: Nuclear localizations of the herpes simplex virus type 1 tegument proteins VP13/14, vhs and VP16 precedeVP22-dependent microtubule reorganization and VP22 nuclear import. J Virol 79: 4730-4743, 2005.

19. Shi NQ, Qi XR, Xiang B and Zhang Y: A survey on 'Trojan Horse' peptides: Opportunities, issues and controlled entry to 'Troy'. J Control Release 194: 53-70, 2014.

20. Yu X, Xu Z, Lei J, Li T and Wang Y: VP22 mediates intercellular trafficking and enhances the in vitro antitumor activity of PTEN. Mol Med Rep 12: 1286-1290, 2015. 